\title{
Detection of Iron Oxide Nanoparticles for Local Chemotherapeutic Treatment Employing Coded Magnetomotive Ultrasound
}

\author{
M.Fink $^{1}$, H. Ermert ${ }^{1}$, C. Alexiou ${ }^{2}$, S.Lyer $^{2}$ \\ ${ }^{1}$ Friedrich-Alexander-University Erlangen-Nuremberg, Department of Sensor Technology, \\ Paul-Gordan-Str. 3/5, 91052 Erlangen, Germany, \\ 2 Universitätsklinikum Erlangen, Department of Otorhinolaryngology, Head and Neck Surgery, Section \\ of Experimental Oncology and Nanomedicine (SEON), Else-Kröner-Fresenius Stiftung-Professorship, \\ Glückstr. 10a, 91054 Erlangen, Germany \\ michael.fink@fau.de
}

\begin{abstract}
Research in biomedical nanotechnology led already to a variety of applications of nanoparticles in diagnosis as well as in therapy. One of these medical applications is Magnetic Drug Targeting (MDT), a cancer treatment technique that allows local chemotherapy of cancerous tissue. For this purpose, chemotherapeutic drugs are bound to magnetic nanoparticles and are accumulated in the tumor region by external magnetic fields. Magnetic nanoparticles can indirectly serve as ultrasound contrast agents. Thus, sonographic technologies can be used as visualization technique for MDT. As nanoparticles are not visible directly using ultrasound imaging techniques due to their weak backscattering, the sonographic detection of nanoparticles has to be attributed to the detection of tissue movements, due to magnetically evoked nanoparticle movements. In this context, a sinusoidal magnetic excitation field is disadvantageous in terms of distinctness of particle induced movements, as naturally occurring movements may match the magnetic field frequency and may lead to mismeasurements. Thus, in this contribution the use of coded magnetic excitation signals, which own a major recognition value, is investigated. Here, frequency coded signals are used as magnetic excitation signals, whereat the frequency modulation is carried out with a Barker sequence offering outstanding correlation properties. We show that coded magnetic fields enable the detection of magnetic nanoparticles even if further tissue movements are superposed.
\end{abstract}

Key words: Magnetic Drug Targeting, Magnetic Nanoparticles, Ultrasound Imaging, Magnetomotive Ultrasound

\section{Introduction}

Magnetic nanoparticles offer several possibilities in medical diagnosis as well as in therapy. One of these medical applications is Magnetic Drug Targeting (MDT), a promising new cancer treatment technique, which enables local chemotherapy. In comparison to traditional chemotherapeutic treatments, MDT allows a reduction of the overall dosage of cytostatic drugs, resulting in reduced side-effects in patients. It is worth mentioning, that at the same time an increased dosage of chemotherapeutic agent in the tumor area can be achieved [1] For this purpose, chemotherapeutic drugs are bound to magnetic nanoparticles and are applied intra-arterially in the vicinity of the tumor. These particles and thereby the medical agent can be accumulated in the tumor area by means of an external static magnetic field. In the currently most extensive preclinical animal study for MDT a high efficiency could be shown recently [2].

A successful MDT treatment depends on the presence of magnetic nanoparticles with an increased density in the tumor area and therefore among others on the position of the magnet, which generates the static magnetic field and which is considerably responsible for the particle distribution. Thus, the visualization of nanoparticles or particle loaded tissue at least is of vital importance in order to optimize the position of the magnet. There are several modes to detect magnetic nanoparticles that are already in clinical use, such as Magnetic Resonance Imaging (MRI). MRI is a well-known technique and is used as standard imaging technique in clinics. There are also imaging techniques under development that are able to 
visualize the nanoparticle loaded tissue, such as Magnetic Particle Imaging (MPI). For this new imaging technique superparamagnetic nanoparticles are used as contrast agents [3]. Both techniques have in common that they need relatively large technical equipment and are very expensive. In contrast, ultrasound is a widespread and rather low cost imaging technology. Therefore, it is desirable to enable the sonographic detection of nanoparticles. However, the size of nanoparticles is challenging ultrasound based detection, as they are not visible directly due to their weak backscattering. Fig. 1 shows the B-mode image of a tissue mimicking phantom which contains nanoparticle loaded tissue. As can be seen, the particle loaded tissue cannot be identified using standard ultrasound imaging techniques.

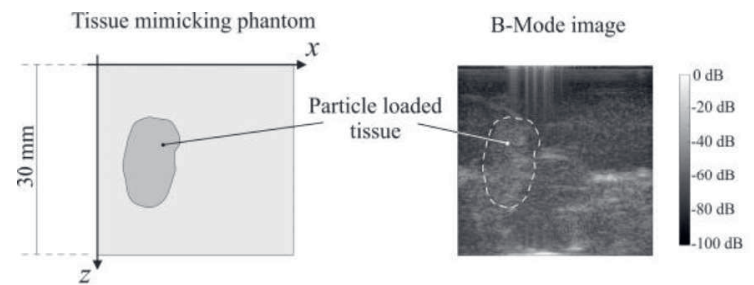

Fig. 1. Schematic image of a tissue mimicking phantom which contains nanoparticle loaded tissue (left) and its B-mode image (right).

Nevertheless, it has been demonstrated that ultrasound imaging techniques can be exploited to detect nanoparticle loaded tissue [4]. This sonographic detection of magnetic nanoparticles is known as Magnetomotive Ultrasound (MMUS). MMUS is based on nanoparticle movement excitations by means of an external time variable magnetic field $\vec{B}(\vec{r}, t)$, which leads to a time variable magnetic force $\vec{F}(\vec{r}, t)$. As the particles are moving due to the time variant magnetic force, the surrounding tissue is moving as well forced by the nanoparticles. The detection of this tissue movement is the aim of MMUS. Conventional ultrasound imaging techniques have been utilized to visualize the resulting tissue movement, such as Doppler technique [4] or Mmode technique [5]. Different modes of movement excitations have been analyzed, such as sinusoidal magnetic fields [6] or pulsed magnetic fields [7]. Furthermore, different evaluation algorithms have been analyzed [8], [9]. In this contribution, the use of coded magnetic excitation fields is investigated.

The paper is organized as follows. First of all the common mono-frequency MMUS algorithm is described. Afterwards the coded MMUS algorithm is introduced and its benefits are discussed. In a further step, the required signal processing steps and the measurement setup to verify the improved detectability of magnetic nanoparticles are presented followed by a conclusion and a short outlook.

It is worth noting that in the following, according to Fig. 1, the axial direction corresponds to the $z$-axis, the lateral direction corresponds to the $x$ axis. Furthermore, the elevational direction points into the plane of projection.

\section{Mono-Frequency Magnetomotive Ultrasound}

Commonly, a sinusoidal field is used as magnetic excitation field in MMUS applications. The sinusoidal excitation leads to a sinusoidal magnetic force if nonlinear magnetic behavior is neglected. It is worth mentioning that a DC offset of half the amplitude of the excitation signal has to be applied, otherwise the magnetic force owns twice the frequency of the magnetic field frequency [10].

The nanoparticles and the surrounding tissue are moving due to the alternating force and, as the setting is observed sonographically, this leads to variations of the collected ultrasound data. If the observed tissue contains magnetic nanoparticles, the excitation signal can be regained from the amplitude of the collected high frequency ultrasound data (rf-data) $h(z, x, t)$ or its phase $\varphi(z, x, t)$. The evaluation of the phase signal with respect to time in a certain point $\left[z_{0}, x_{0}\right]$ of the ultrasound image in the frequency domain enables the detection of tissue oscillations in this image point.
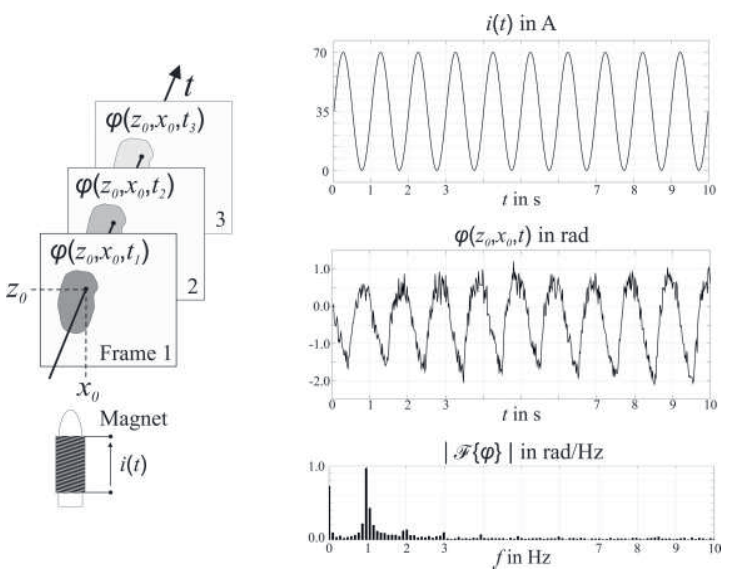

Fig. 2. Variation of the phase of the rf-data in a certain image point inside the particle loaded tissue.

Since the particles oscillate with the magnetic field frequency, this enables indirectly the detection of the presence of magnetic 
nanoparticles. As can be seen from Fig. 2 the magnetic field frequency $f_{l}$ is mapped onto the phase signal of the rf-data, if $\left[z_{0}, x_{0}\right]$ is located in the particle loaded tissue. The appropriate spectral component of the phase signal can therefore be regarded as an indicator for the presence of magnetic nanoparticles. Thereto, the magnetic field frequency must not match the frequency of naturally occurring tissue movements due to respiration or heartbeat. As these biological processes are commonly located at low frequencies up to approximately $5 \mathrm{~Hz}$, the magnetic field frequency has to exceed this frequency range.

However, magnets that are optimized for accumulating magnetic particles or for MDT applications, respectively, are predominantly designed for static magnetic fields. Nevertheless, with a view to clinical applicability it is desirable to utilize the same magnet for the visualization as well as for the accumulation of the nanoparticles. But due to eddy-current losses the reclaimable frequency range of these magnets is restricted to low frequencies and unfortunately matches the spectral range of several biological processes. To be able to draw a distinction between naturally occurring tissue movements and particle induced movements of the same frequency, it can be considered that particle induced movements have to match the phase of the magnetic excitation field [6]. However, due to phaseambiguity of sinusoidal signals such a phasetracking algorithm only yields poor improvement [10]. Thus, with a view to practical application a sinusoidal excitation is unfavorable. Therefore, in this contribution the use of a frequency coded signal as magnetic excitation signal is investigated, which promises an improved detectability and separability from other sources of tissue movement.

\section{Coded Magnetomotive Ultrasound}

Since a pure sinusoidal signal as magnetic excitation signal is disadvantageous for MMUS detection of magnetic nanoparticles, alternative excitation signals are required. In this contribution a frequency modulated signal serves as magnetic excitation signal and has to be decoded from the collected rf-data $h(z, x, t)$, by evaluating its phase $\varphi(z, x, t)[10]$.

Coded excitation signals are advantageous due to their distinct recognition value. Usually, the generation of coded excitation signals is based on code sequences featuring two possible states, i.e., -1 and +1 [11]. In this contribution, -1 and +1 correspond to the magnetic field frequencies $f_{1}$ and $f_{2}$. Fig. 3 depicts both, the employed 4-bit Barker code sequence and the excitation signal. Furthermore, Fig. 3 shows the phase signal with respect to time in an exemplarily chosen image point within the particle loaded tissue. In comparison to the mono-frequency case, in the coded MMUS case the spectrum of the phase signal is broadband. Nevertheless, the amplitude spectrum owns a distinct peak at $f_{l}$. Thus, the presence of this spectral component still can be regarded as an indicator for the presence of magnetic nanoparticles.
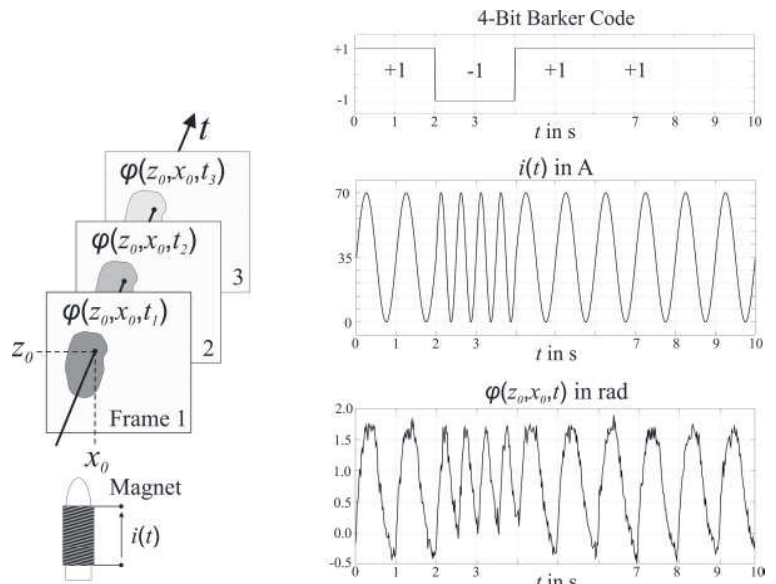

$\varphi\left(z_{0}, x_{0}, t\right)$ in rad

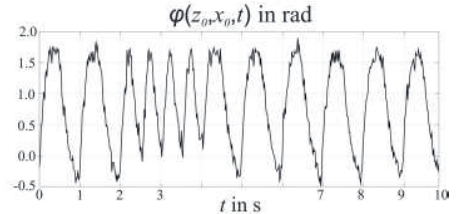

$|\mathscr{F}\{\varphi\}|$ in $\mathrm{rad} / \mathrm{Hz}$

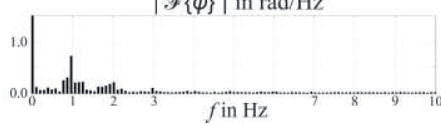

Fig. 3. 4-Bit Barker code sequence, excitation signal and variation of the phase of the rf-data in an image point inside the particle loaded tissue.

\section{Signal Processing}

Since the acquired signals are time discrete, the signal processing has to be performed in the time discrete domain. However, to simplify further explanations, the signal processing is given for time continuous signals.

In this contribution a frequency modulated signal serves as magnetic excitation signal and has to be decoded from the collected rf-data $h(z, x, t)$. For this purpose, for each image point $\left[z_{i}, x_{j}\right]$ we employ three signal processing steps:

(i) calculation of the phase $\varphi\left(z_{i}, x_{j}, t\right)$ from the rfdata via Hilbert transform $\mathcal{H}\{\bullet\}$

$$
\varphi\left(z_{i}, x_{j}, t\right)=\arg \left[\mathrm{H}\left\{h\left(z_{i}, x_{j}, t\right)\right\}\right],
$$

(ii) evaluation of the phase signal in the frequency domain

$$
\Phi\left(z_{i}, x_{j}, f\right)=\int_{-\infty}^{+\infty} \varphi\left(z_{i}, x_{j}, t\right) e^{-j 2 \pi f t} \mathrm{~d} t
$$


(iii) cross-correlation for retrieving image points, in which the tissue oscillates time delayed to the magnetic excitation field

$$
R\left(z_{i}, x_{j}, \tau\right)=\frac{1}{T} \int_{-T / 2}^{+T / 2} \varphi\left(z_{i}, x_{j}, t\right) u(t+\tau) \mathrm{d} t,
$$

with $u(t)$ the magnetic excitation signal. Image points, in which the tissue oscillates time delayed to the excitation signal will not be considered anymore. The latter signal processing step can be seen equivalent to the phase-tracking algorithm of mono-frequency MMUS. However, in comparison to the phasetracking algorithm, coded sequences do not suffer from phase-ambiguity and, thus, crosscorrelation based filtering of coded sequences works more effective. Each point $\left[z_{i}, x_{j}\right]$ in the ultrasound image is investigated whether

(i) the utilized code sequence exists in the phase signal $\varphi\left(z_{i}, x_{j}, t\right)$,

(ii) the temporal relation between the possibly retrieved code sequence and the excitation signal $u(t)$ is correct.

As a result of these conditions, a mask $M(z, x)$ can be shaped, which filters out all image points that do not satisfy these conditions. If for a certain image point $\left[z_{i}, x_{j}\right]$ the used code sequence cannot be found in the phase signal $\varphi\left(z_{i}, x_{j}, t\right)$ or if the code sequence is time delayed to the magnetic field, the appropriate value of $M\left(z_{i}, x_{j}\right)$ is set equal to zero. Otherwise $M\left(z_{i}, x_{j}\right)$ is set equal to one.

The previously calculated amplitude spectrum can be evaluated at the frequency $f_{l}$ and can then be filtered via the mask $M(z, x)$. The resulting image $\Gamma(z, x)$ then follows from the element-wise multiplication of the amplitude spectrum at $f_{l}$ and the mask $M(z, x)$

$$
\Gamma\left(z_{i}, x_{j}\right)=\Phi\left(z_{i}, x_{j}, f_{1}\right) M\left(z_{i}, x_{j}\right) .
$$

\section{Measurement Setup}

The measurement setup to verify the ability for identification of particle loaded tissue via coded MMUS consists of a high field gradient magnet [12], an ultrasound unit and a tissue mimicking phantom. The magnet was used to induce an alternating magnetic field. However, in consideration of MDT the magnet was primarily designed for static magnetic fields. Alternating magnetic fields lead to eddy current losses. Thus, a water cooling system is used to control the temperature of the electromagnet. All measurements were performed using the ultrasound system Ultrasonix TOUCH. A linear array (Ultrasonix L9-4/48) was applied as ultrasound transducer. In order to detect vibrations inside the observed tissue or inside the tissue mimicking phantom, respectively, a sequence of ultrasound images was recorded at a framerate of $55 \mathrm{~Hz}$. Each frame has a size of 1824 (axial direction) x 256 (lateral direction) pixels. The tissue mimicking phantom was made out of polyvinyl alcohol, which is suitable to be used as tissue mimicking material in medical ultrasound applications [13]. The tissue mimicking phantom is located in between the ultrasound transducer and the magnet. It contains a latex bubble with nanoparticle loaded tissue, whereat the diameter of the particle loaded tissue is approximately $10 \mathrm{~mm}$. The distance $d_{1}$ between magnet pole tip and latex bubble is $5 \mathrm{~mm}$, while the distance $d_{2}$ between ultrasound transducer and latex bubble is $15 \mathrm{~mm}$.

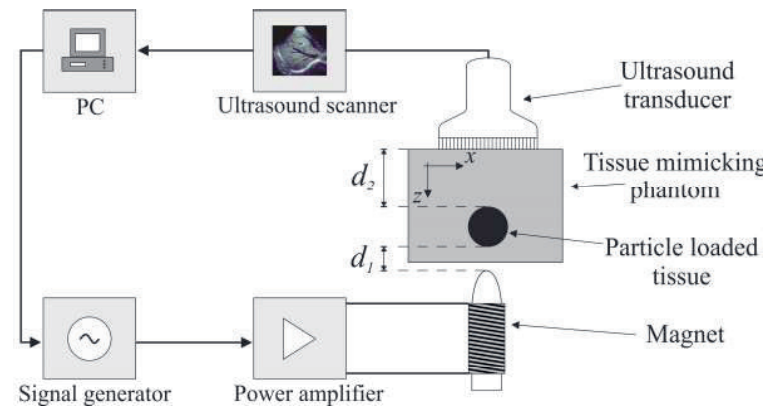

Fig. 4. Measurement setup.

The measurement setup can be seen in Fig. 4 . The collected high frequency ultrasound data were postprocessed using the algorithm described before.

\section{Results}

With a view to verifying the presented MMUS approach, we investigate the detectability of particle loaded tissue in case of further tissue movements that are superposed. The induced noise signal is randomly distributed in the frequency range up to $5 \mathrm{~Hz}$, while its RMS value can be chosen arbitrarily.

Thus, the mono-frequency MMUS algorithm and the coded MMUS algorithm can be investigated at different signal-to-noise ratios (SNR) and their performance can be compared. Firstly, filtering via the mask $M(z, x)$ is shown without noise signal. Therefore, a frequency coded 4-bit Barker sequence as described before is used. The frequencies $f_{1}$ and $f_{2}$ were chosen to be $1 \mathrm{~Hz}$ and $2 \mathrm{~Hz}$, respectively. In the mono-frequency MMUS case the magnetic field frequency is chosen to be $1 \mathrm{~Hz}$. 

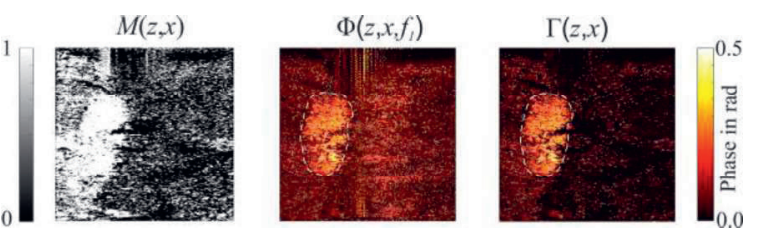

Fig. 5. Cross-correlation based filter (left), amplitude spectrum evaluated at the magnetic field frequency (center) and filtered amplitude spectrum (right). The particle loaded tissue is marked by a dashed line.

Fig. 5 shows the amplitude spectrum $\Phi\left(z, x, f_{l}\right)$ evaluated at $1 \mathrm{~Hz}$ as well as the mask $M(z, x)$ and the filtered amplitude spectrum $\Gamma(z, x)$. As can be seen from Fig. 5 the cross-correlation based filter leads to a significant improvement referring to identification of nanoparticle loaded tissue.

Subsequently, noise signal shall be superposed, whereat the effect of different signal-to-noise ratios is investigated. It is worth noting that phase-tracking is already implemented in $\Phi\left(z, x, f_{1}\right)$, so, the monofrequency MMUS evaluation is already optimized. Fig. 6 shows the evaluation as described in this contribution while SNR varies from $6 \mathrm{~dB}$ to $-6 \mathrm{~dB}$. It can be clearly seen that coded magnetic excitation signals are favorable in terms of spatial confinement of the particle loaded tissue. Particularly, at SNR lower than $0 \mathrm{~dB}$ the mono-frequency MMUS algorithm hardly allows the identification of the particle loaded tissue, while the coded MMUS algorithm is still capable to do so.

\section{Conclusion}

This study shows a comparison between the classical mono-frequency MMUS algorithm and the coded MMUS algorithm. It could be shown that coded magnetic excitation fields are advantageous in terms of spatial confinement of the particle loaded tissue. Especially in case of superposed tissue movements coded fields lead to an improved detectability of the particle loaded tissue. In real applications superposed tissue movements are inevitable as respiratory movements or heartbeat movements always are present.

As the measurements on tissue mimicking phantoms show promising results, in the next step measurements applied to real biological tissue perfused by magnetic nanoparticles will be considered.
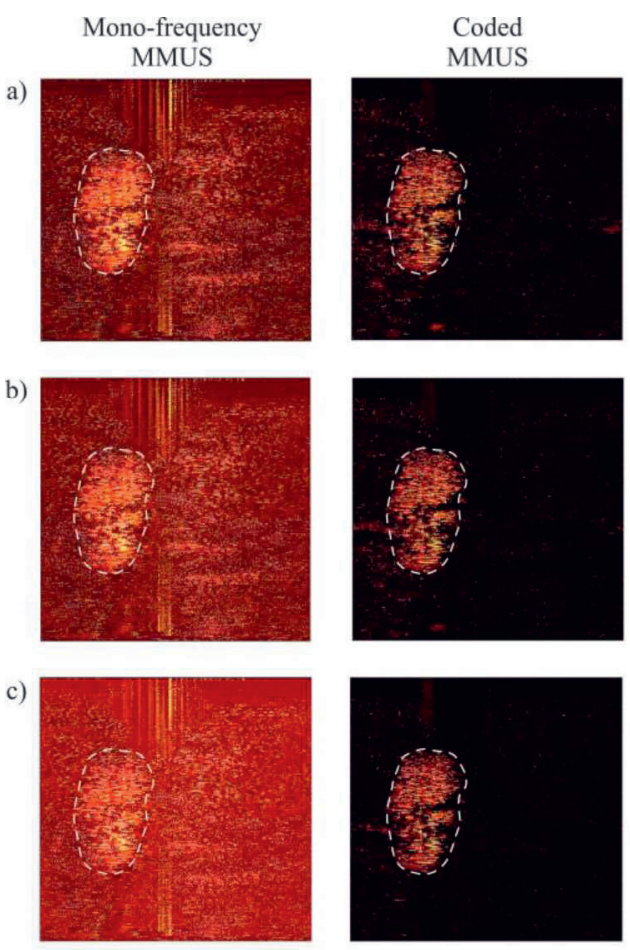

d)
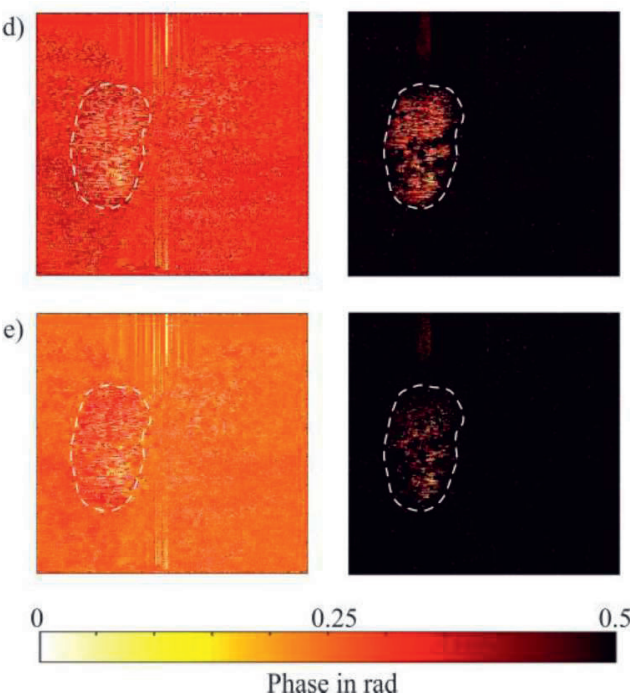

Fig. 6. Mono-frequency MMUS exploiting the phase-tracking algorithm (left) and coded MMUS (right) at different signal-to-noise ratios of $6 \mathrm{~dB}$ (a), $3 d B$ (b), $0 d B$ (c), $-3 d B$ (d) and $-6 d B$ (e). The particle loaded tissue is marked by a dashed line.

\section{Aknowledgements}

This study was supported by the German Research Foundation (DFG) (AL552/8-1).

\section{References}

[1] C. Alexiou, A. Schmidt, R. Klein, P. Hulin, C. Bergemann and W. Arnold, Magnetic Drug Targeting: Biodistribution and Dependency on Magnetic Field Strength, Journal of Magnetism and Magnetic Materials 252, 363-366 (2002); doi: $10.3109 / 1061186031000150791$ 
[2] R. Tietze, S. Lyer, S. Dürr, T. Struffert, T. Engelhorn, M. Schwarz, E. Eckert, T. Göen, S. Vasylyev, W. Peuckert, F. Wieckhorst, L. Trahms, A. Dörfler and C. Alexiou, Efficient Drug-Delivery Using Magnetic Nanoparticles - Biodistribution and Therapeutic Effects in Tumor Bearing Rabbits, Nanomedicine: Nanotechnology, Biology and Medicine 9, 961-971 (2013); doi: 10.1016/j.nano.2013.05.001

[3] K. Lüdtke-Buzug, J. Haegele, S. Biederer, T. Sattel, M. Erbe, R. Duschka, J. Barkhausen and F. Vogt, Comparison of Commercial Iron Oxide Based MRI Contrast Agents with Synthesized Highperformance MPI Tracers, Biomedical Engineering 8, 527-533 (2013); doi: 10.1515/bmt2012-0059

[4] J. Oh, M. Feldmann, J. Kim, C. Condit, S. Emilianov and T. Miller, Detection of Magnetic Nanoparticles in Tissue Using Magneto-Motive Ultrasound, Nanotechnology 17, 4183-4190 (2006); doi: 10.1088/0957-4484/17/16/031

[5] M. Mehrmohammadi, J. Oh, L. Ma, E. Yantsen, T. Larson, S. Malidi, S. Park, K. Johnston, K. Sokolov, T. Milner and S. Emilianov, Imaging of Iron Oxide Nanoparticles Using Magneto-Motive Ultrasound, Proc. IEEE IUS 2007, 652-655 (2007); doi: 10.1109/ULTSYM.2007.169

[6] M. Evertsson, M. Cinthio, S. Fredriksson, F. Olsson, H. Persson and T. Jansson, PhaseLocked Magnetomotive Ultrasound Imaging of Superparamagnetic Iron-Oxide Nanoparticles, Proc. IEEE IUS 2010, 1007-1010 (2010); doi: 10.1109/ULTSYM.2010.5935431

[7] M. Mehrmohammadi, J. Oh, S. Aglyamov, A. Karpiouk and S. Emilianov, Pulsed Magneto-
Acoustic Imaging, Proc. IEEE EMBS 2009, 4771 4774 (2009); doi: 10.1109/IEMBS.2009.5334214

[8] M. Fink, H. Ermert, M. Löffler, A. Sutor, B. Tewes, A. Koch, C. Alexiou and S. Lyer, Sonographic Detection of Nanoparticles Uses for Magnetic Drug Targeting, Proc. Sensor 2015, 430-435 (2015); doi: 10.5162/sensor2015/C5.3

[9] M. Fink, M. Nüsslein, H. Ermert, C. Alexiou and S. Lyer, Sonographic Detection of Magnetic Nanoparticles for Magnetic Drug Targeting in Weak Echogenic Tissue, Proc. IEEE IUS 2015, (2015); doi: 10.1109/ULTSYM.2015.0263

[10] M. Fink, H. Ermert, S. Lyer and C. Alexiou, Sonographic Detection of Magnetic Nanoparticles for Magnetic Drug Targeting Using Coded Magnetic Fields, Proc. IEEE IUS 2016 (2016); doi: 10.1109/ULTSYM.2016.7728404

[11] Q. Peng and L. Zhang, High-Resolution Ultrasound Displacement Measurement Using Coded Excitations, IEEE Transactions on Ultrasonics, Ferroelectrics, and Frequency Control 58, no.1, 122-133 (2011); doi: 10.1109/TUFFC.2011.1779

[12] C. Alexiou, D. Diehl, P. Henninger, H. Iro, R. Roecklein, W. Schmidt and H. Weber, A High Field Gradient Magnet for Magnetic Drug Targeting, IEEE Transactions on Applied Superconductivity 16, no.2, 1527-1530 (2006); doi: 10.1109/TASC.2005.864457

[13] K.J.M. Surry, H.J.B. Austin, A. Fenster and T.M. Peters, Poly (Vinyl Alcohol) Cryogel Phantoms for Use in Ultrasound and MR Imaging, Physics in Medicine and Biology 49, no.24, 5529-5546 (2004); doi: 10.1088/0031-9155/49/24/009 